\title{
Fluoroquinolone and no risk of Achilles-tendinopathy in childhood pneumonia under eight years of age - a nationwide retrospective cohort
}

\author{
Yunsun Kim ${ }^{1 \#}$, Gun Woo Park ${ }^{2 \#}$, Sangyoung Kim ${ }^{1}$, Hui Jeong Moon ${ }^{1}$, Sungho Won ${ }^{2,3,4}$, Wankyo Chung ${ }^{2}$, \\ Hyeon-Jong Yang ${ }^{1,5} \wedge$
}

${ }^{1}$ SCH Biomedical Informatics Research Unit, Soonchunhyang University Seoul Hospital, Seoul, Korea; ${ }^{2}$ Department of Public Health Sciences, Graduate School of Public Health, Seoul National University, Seoul, Korea; ${ }^{3}$ Interdisciplinary Program of Bioinformatics, Seoul National University, Seoul, Korea; ${ }^{4}$ Institute of Health and Environment, Seoul National University, Seoul, Korea; ${ }^{5}$ Pediatric Allergy and Respiratory Center, Department of Pediatrics, Soonchunhyang University Seoul Hospital, Soonchunhyang University College of Medicine, Seoul, Korea

Contributions: (I) Conception and design: Y Kim, GW Park, W Chung, HJ Yang; (II) Administrative support: HJ Yang, S Won; (III) Provision of study materials or patients: Y Kim, GW Park, S Kim, HJ Moon; (IV) Collection and assembly of data: Y Kim, GW Park, S Kim, HJ Moon; (V) Data analysis and interpretation: Y Kim, GW Park, S Won, W Chung, HJ Yang; (VI) Manuscript writing: All authors; (VII) Final approval of manuscript: All authors.

\#These authors contributed equally to this work.

Correspondence to: Hyeon-Jong Yang, MD, PhD. Department of Pediatrics, Soonchunhyang University Seoul Hospital, Soonchunhyang University College of Medicine, 59 Daesagwan-ro, Yongsan-gu, Seoul, 04401, Korea. Email: pedyang@schmc.ac.kr; Wankyo Chung, PhD. Department of Public Health Science, Graduate School of Public Health, Seoul National University, 1 Kwanak-ro Kwanak-gu Seoul 08826, Korea. Email: wankyo@snu.ac.kr.

Background: The emergence of macrolide-resistant Mycoplasma pneumoniae pneumonia (MRMP) has made its treatment challenging. A few guidelines have recommended fluoroquinolones (FQs) as secondline drugs of choice for treating MRMP in children under the age of eight, but concerns about potential adverse events (i.e., Achilles tendinopathy; AT) have been raised. The aim of this study was to investigate the relationship between the use of FQs and the risk of AT in pneumonia in children under eight years of age.

Methods: Children hospitalized with pneumonia (total of 2,213,807 episodes) from 2002 to 2017 were enrolled utilizing the Korean National Health Insurance Sharing Service (NHISS) database. The independent risk of FQs for AT was analyzed by a generalized estimating equation with adjustment for age, sex, and underlying diseases.

Results: Among 2,213,807 episodes of pneumonia hospitalization, children in a total of 6,229 episodes $(0.28 \%$ ) were treated with FQs (levofloxacin $40.9 \%$, ciprofloxacin $36.1 \%$, moxifloxacin $11.6 \%$, and others 11.4\%). The FQ-exposure group showed a $0.19 \%(12 / 6,229)$ incidence of AT within 30 days after the first administration of FQ. The use of FQs increased the risk of AT (OR 3.00; 95\% CI: 1.71-5.29), but became null after adjusting for age, sex, and underlying diseases (aOR 0.85; 95\% CI: 0.48-1.51). All AT related to the use of FQs occurred after the use of ciprofloxacin or levofloxacin, and not in children under eight years of age. Conclusions: AT was a rare adverse event of FQ use for childhood pneumonia, particularly under eight years of age. Clinicians could consider using FQs as a second-line option in the treatment of childhood pneumonia when there are no alternative therapeutic options.

Keywords: Fluoroquinolones (FQs); Achilles tendon; tendinopathy; pneumonia; child

Submitted Jun 19, 2020. Accepted for publication Apr 14, 2021.

doi: $10.21037 /$ jtd-20-2256

View this article at: http://dx.doi.org/10.21037/jtd-20-2256

$\wedge$ ORCID: 0000-0002-7287-4300. 


\section{Introduction}

Community-acquired pneumonia (CAP) remains a leading cause of morbidity and mortality worldwide. Streptococcus Pneumoniae is the most prevalent cause of CAP in most countries. However, increasing numbers of Mycoplasma pneumoniae pneumonia (MPP) have been recognized, especially in children and young adults. MPP causes $40 \%$ or more of the CAP and as many as $18 \%$ of the cases in children requiring hospitalization (1).

M. pneumoniae lacks cell walls. Therefore, it is resistant to beta-lactams and all antimicrobials targeting the cell wall. Macrolides have long been regarded as the first-line treatments for MPP, mainly due to their low minimum inhibitory concentration and safety in children. However, the prevalence of macrolide-resistant Mycoplasma pneumoniae pneumonia (MRMP) has abruptly increased in recent years (2), particularly in East Asian countries, such as China, Japan, and Korea, with high macrolide pressure (3). This is evident by the highest macrolide resistance rates reported in countries with extensive macrolide use. However, the rate decreased by $43.6 \%$ in 2015 from its highest rate of $81.6 \%$ in 2012, along with a reduction in macrolide prescriptions in Japan (4).

The emergence of MRMP has made its treatment challenging. A few guidelines have recommended fluoroquinolones (FQs) as the second-line drugs of choice for treating MRMP in children (5,6), but concerns about its potential adverse events (i.e., Achilles tendinopathy; AT) have been raised. Therefore, recommendations for the use of FQs according to age still conflict among the guidelines. Although MPP is usually a mild, self-limited disease with a good response to macrolides, it can cause various extra-pulmonary complications and progress to severe life-threatening conditions. Clinicians should be aware of macrolide treatment failure and consider using alternative drugs if symptoms persist or if there are signs of clinical deterioration. Delayed decisions to use secondline antimicrobials may induce further complications, as well as a healthcare-related burden. The aim of this study was to investigate the relationship between the use of FQs and the risk of AT in children under eight years of age with pneumonia.

We present the following article in accordance with the STROBE reporting checklist (available at http://dx.doi. org/10.21037/jtd-20-2256).

\section{Methods}

\section{Data source}

The National Health Insurance Service (NHIS), a government-affiliated agency responsible for examining the accuracy of claims for the National Health Insurance and National Medical Aid in Korea, covers approximately $96.6 \%$ of the Korean population (7). The National Health Insurance Sharing Service (NHISS) provided by NHIS conducts reviews and assessments of all medical insurance claims in South Korea. The NHISS database includes information about demographics (age and sex), medical healthcare service data, including diagnostic codes (International Statistical Classification of Disease and Related Health Problems $10^{\text {th }}$ Edition, ICD-10), dispensed or prescribed medications, and diagnostic tests and procedures. Preferably, it contains information on healthcare utilization at hospitals, emergency departments, outpatient clinics, and dispensed medication at pharmacies. There were missing or outlying values in key fields, such as drug names, quantities, date dispensed, and duration in less than $0.5 \%$ of all records. We used the NHISS data to identify the effects of FQs in the occurrence of AT in children with pneumonia under eight years of age.

\section{Study population and design}

We constructed a nationwide, population-based retrospective cohort of community-acquired childhood pneumonia (patients under 19 years of age) from 2002 to 2017 in Korea. We identified a total of 1,418,541 children hospitalized with pneumonia during the study period. We excluded individuals with missing or outlying values in demographic variables, such as birth year, birth quarter, sex, and age. We also excluded data from 2002 and 2017 to avoid the underdetection of AT or the use of FQs in the first and last year of the study period. As a result, we ended up with 2,213,807 pneumonia episodes requiring hospitalization. Pneumonia-related hospitalization was defined when a pneumonia diagnosis (J11-18 as the primary or first additional diagnosis) was made at the time of hospitalization.

\section{Definitions of dependent and independent variables}

AT was considered a dependent variable. For each child, 
we checked whether he/she received an AT diagnostic code [(S86.08 and M76.6) and no S86.00] as the primary or first additional diagnosis within 30 days after the first administration of FQs. In the case of multiple episodes of AT, a six-month window was applied to ensure the independence of the episode.

FQ use was defined as the main independent variable when the drug code (i.e., J01MA) was observed at the time of hospitalization. For each subject, cumulative duration (days) and age at use were recorded and used for subgroup analyses. Detailed information on the FQs is described in Table S1.

For the severity of pneumonia, we considered any case of chest computed tomography, oxygen administration, intensive care unit admission, intubation, ventilator care or cardiopulmonary resuscitation during the same hospitalization periods (detailed information is described in Table S2). Other potential covariates including underlying diseases, such as cardiovascular diseases, congenital respiratory diseases, neuromuscular diseases, genetic metabolic diseases, immunodeficiency, neoplasms, autoimmune disease, and transplantation within 12 months before the first administration of FQs, were included in the statistical model. Table S3 presents the detailed information.

\section{Etbical considerations}

The study was conducted in accordance with the Declaration of Helsinki (as revised in 2013). The research protocol for this study was approved by the Ethical Review Board of Soonchunhyang University Seoul Hospital (approval number: SCHUH 2017-06-006). The requirement for informed consent was waived due to the retrospective nature of the study. The authors are accountable for all aspects of the work (if applied, including full data access, integrity of the data and the accuracy of the data analysis) in ensuring that questions related to the accuracy or integrity of any part of the work are appropriately investigated and resolved.

\section{Statistical analysis}

We investigated the relationship between the use of FQs and the risk of AT using generalized estimation equation (GEE) models at the individual level. The GEE model was adjusted for sex, age group, pneumonia severity, and underlying diseases. AT was considered a dependent variable, and the logit link function was used. The correlation between the longitudinally observed dependent variables of each subject was adjusted using sandwich estimators. In addition, potential confounders were adjusted for sex, age group, the index of severity, and the index of other diseases. We presented a crude model and an adjusted model to estimate the odds ratios (ORs) and 95\% confidence intervals (CIs), respectively. Quasi-Akaike's Information Criteria (QIC) were used to select the optimal model from among several options and the predicted values were calculated using the selected model. In subgroup analyses, we further restricted the cohort groups according to the subtypes of FQs, age group, and the duration of FQ use. All statistical analyses were conducted using the statistical computing program, SAS Enterprise Guide software (version 7.1).

\section{Results}

The characteristics of the study population are presented in Table 1. Among 2,213,807 episodes of childhood pneumonia requiring hospitalization, children in a total of $6,229(0.28 \%)$ episodes were given FQs for the treatment of pneumonia. Most of the cases were under eight years of age $(87.79 \%)$. The mean age was $3.31 \pm 3.66$ years and $53.74 \%$ of the total study population was boys. There were $1,943,555$ episodes of hospitalizations for CAP in children under eight years of age and the numbers decreased with increasing age. Approximately 3.75\% of the episodes met the operational diagnosis of severe pneumonia. The most common preexisting diseases were cardiovascular diseases (3.49\%), followed by genetic metabolic diseases $(1.18 \%)$, neuromuscular diseases $(0.79 \%)$, neoplasms $(0.24 \%)$, congenital respiratory diseases $(0.16 \%)$, immunodeficiency $(0.08 \%)$, autoimmune disease $(0.07 \%)$, and transplantation $(0.05 \%)$. A total of 1,429 cases of Achilles tendinitis was observed among the 2,213,807 CAP episodes (0.06\%), but no Achilles tendon rupture was observed.

Table 2 shows the characteristics of the subpopulation exposed to FQs. Among 6,229 exposures to FQs, levofloxacin (40.9\%) and ciprofloxacin (36.1\%) were the most common. The total duration of FQ use was $8.50 \pm 5.95$ days. Contrary to the age distribution in Table 1, most cases here were in children over 12 years of age.

Table 3 shows the GEE model results for the use of FQs on AT. The FQ-exposure group showed a 0.19\% (12/6,229) incidence of AT, and a 3-fold higher risk than the nonexposure group (OR 3.00, 95\% CI: 1.71-5.29, $\mathrm{P}<0.0001$ ), but the statistical significance became null after adjusting for age, sex, pneumonia severity, and underlying diseases (aOR 0.85 , 95\% CI: $0.48-1.51, \mathrm{P}=0.57)$. Compared to the group 
Table 1 Demographics of the study population (2,213,807 episodes of childhood pneumonia requiring hospitalization) from 2002 to 2017 in Korea

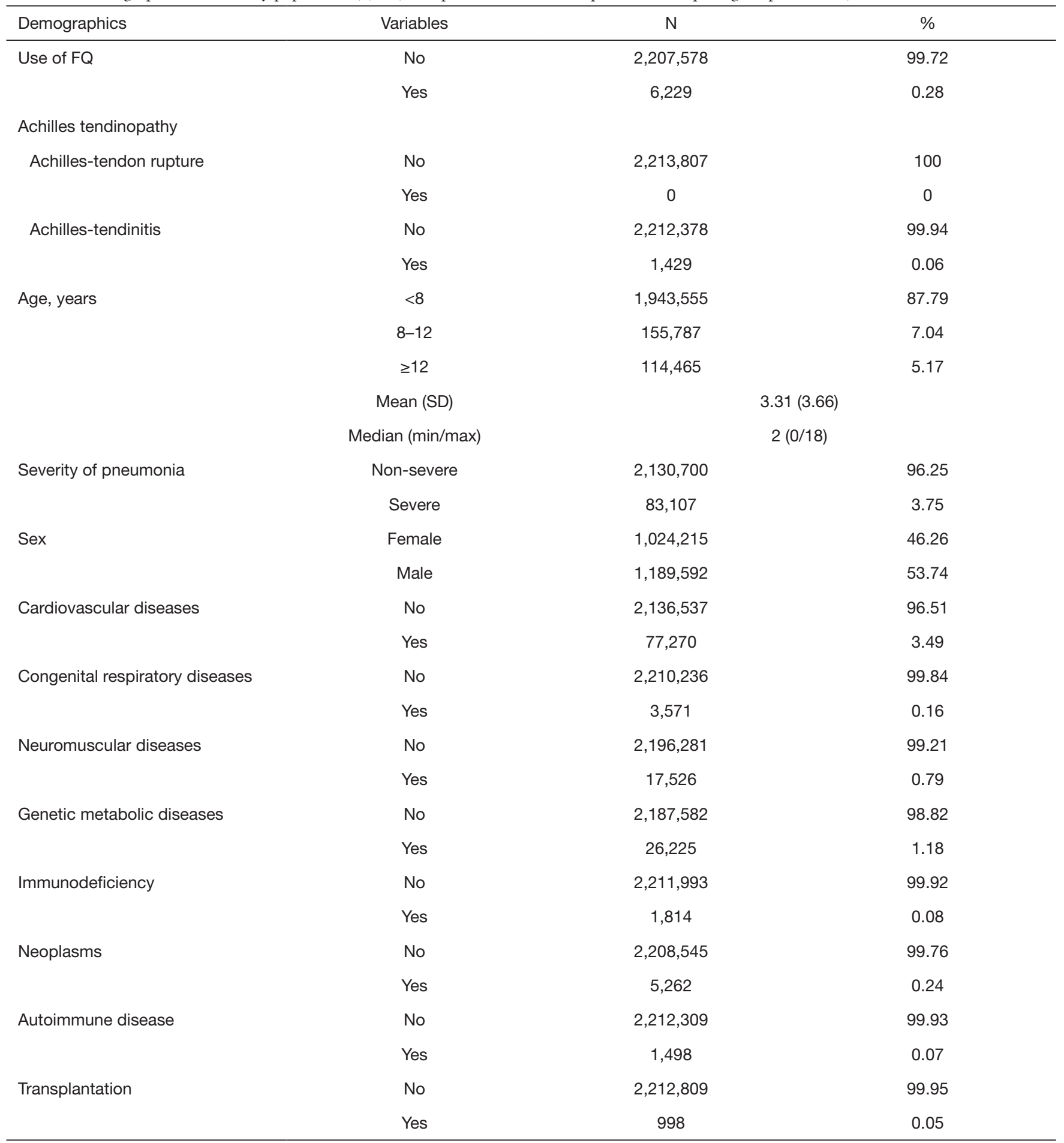


Table 2 Characteristics of the subpopulation exposed to fluoroquinolones (6,229 episodes of childhood pneumonia requiring hospitalization) from 2002 to 2017 in Korea

\begin{tabular}{|c|c|c|}
\hline & $\mathrm{N}$ & $\%$ \\
\hline Levofloxacin & 2,545 & 40.9 \\
\hline Ciprofloxacin & 2,249 & 36.1 \\
\hline Moxifloxacin & 725 & 11.6 \\
\hline Garenoxacin & 0 & 0 \\
\hline Sparfloxacin & 8 & 0.1 \\
\hline Lomefloxacin & 23 & 0.4 \\
\hline Ofloxacin & 351 & 5.6 \\
\hline Duration of use of FQs (day), Mean (SD) & $8.50(5.95)$ & \\
\hline \multicolumn{3}{|l|}{ Age, years } \\
\hline$<8$ & 933 & 14.98 \\
\hline $8-12$ & 501 & 8.04 \\
\hline$\geq 12$ & 4,795 & 76.98 \\
\hline Mean (SD) & \multicolumn{2}{|c|}{$13.87(4.71)$} \\
\hline Median (min/max) & \multicolumn{2}{|c|}{$16(0 / 18)$} \\
\hline
\end{tabular}

12 years old or older, the 8- to 11-year age group showed approximately two-fold higher risk (aOR 1.95, 95\% CI; $1.70-2.24, \mathrm{P}<0.0001$ ), but the group under eight years of age showed 93\% less risk for AT (aOR 0.07, 95\% CI: 0.06-0.09, $\mathrm{P}<0.0001)$. Genetic metabolic diseases and autoimmune diseases increased the risk of AT, independently of the use of FQs (aOR 1.68, 95\% CI: 1.15-2.45) (aOR 2.35, 95\% CI: 1.02-5.4, $\mathrm{P}=0.044)$ after multiple regression analysis.

Table 4 shows the association between the exposure to FQs and the risk of AT according to the subtypes of FQs. Eight cases of AT occurred after exposure to ciprofloxacin $(0.36 \%, 8 / 2,249)$, and four cases occurred after levofloxacin $(0.16 \%, 4 / 2,545)$, but no case occurred after exposure to other FQs. There was no case of AT in children under eight years of age. No duration-dependent response was observed (aOR 0.93, 95\% CI: 0.79-1.1).

\section{Discussion}

In this study, we investigated the use of FQs and the risk of AT in a nationwide childhood pneumonia cohort from 2002 to 2017 and found that the incidence of AT related to the use of FQs was rare $(0.19 \%, 12 / 6,229)$, and most cases of AT were related to underlying diseases, such as genetic metabolic diseases or autoimmune disease, not from the use of FQs alone. In addition, we found no case of AT in children under eight years of age, and no durationdependent response to FQ use.

Emerging resistance to macrolides is of great concern because it creates a therapeutic conundrum, particularly for young children in whom second-line antimicrobials are relatively contraindicated. Recent studies have reported that MRMP showed more rapid progression; and higher extra-pulmonary complications than macrolidesensitive mycoplasma pneumonia $(29.6 \%$ extrapulmonary complications in children with MRMP) (8). Acute respiratory distress syndrome or acute respiratory failure are the most well known and most serious intrapulmonary complications (9). The importance of the early administration of antibiotics for MPP patients was 
Table 3 Generalized estimating equation model for the use of fluoroquinolones on Achilles tendinopathy among Korean children with pneumonia (2,213,807 of episodes) from 2002 to 2017

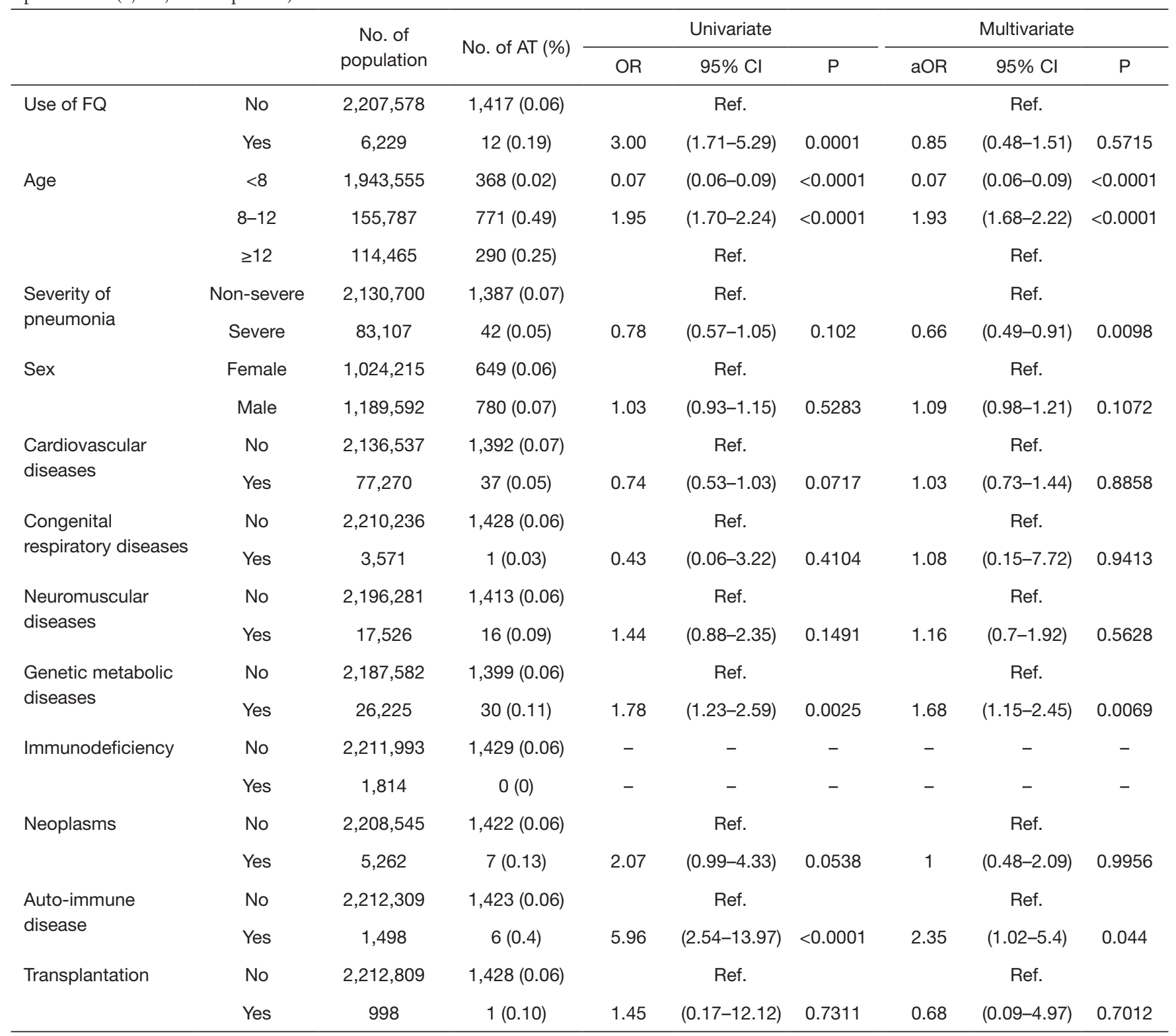

aORs, adjusted for age, severity, sex, cardiovascular diseases, congenital respiratory diseases, neuromuscular diseases, genetic metabolic diseases, neoplasms, autoimmune disease, and transplantation. AT, occurrence of Achilles tendinopathy.

reinforced by a study reporting that the hospital mortality for MP-infected and ICU-admitted patients was 29.4\% (10).

Most guidelines recommend respiratory FQs as first-line therapy for adult CAP (11). FQs act against topoisomerases and inhibit DNA synthesis and replication, and are highly effective against MP in vitro (12). The therapeutic efficacy of FQs has been reported in adolescents and adults with MRMP (13), and also in children (14). The Pediatric
Infectious Diseases Society and the Infectious Diseases Society of America recommend azithromycin as the first-line antibiotic, and clarithromycin, erythromycin, doxycycline (for children aged $\geq 8$ years), levofloxacin or moxifloxacin (for adolescents) as second-line drugs (15), whereas the Japanese guidelines and the 2015 Hong Kong Pediatric CAP guidelines recommend tosufloxacin (in Japan) (5), and levofloxacin (in Hong Kong) (6) as second- 
Table 4 Generalized estimating equation (GEE) model for the subtypes of fluoroquinolones on Achilles tendinopathy Korean children with pneumonia exposed to fluoroquinolones (6,229 episodes) from 2002 to 2017

\begin{tabular}{|c|c|c|c|c|c|c|c|c|c|}
\hline & & $\begin{array}{c}\text { No. of } \\
\text { population }\end{array}$ & AT (\%) & \multicolumn{3}{|c|}{ Univariate } & \multicolumn{3}{|c|}{ Multivariate } \\
\hline \multirow[t]{7}{*}{ FQ subtypes } & Ciprofloxacin & 2,249 & $8(0.36)$ & \multicolumn{3}{|c|}{ Ref. } & \multicolumn{3}{|c|}{ Ref. } \\
\hline & Levofloxacin & 2,545 & $4(0.16)$ & 0.44 & $(0.13-1.46)$ & 0.1809 & 0.49 & (0.14-1.66) & 0.2501 \\
\hline & Moxifloxacin & 725 & 0 & - & - & - & - & - & - \\
\hline & Garenoxacin & 0 & 0 & - & - & - & - & - & - \\
\hline & Sparfloxacin & 8 & 0 & - & - & - & - & - & - \\
\hline & Lomefloxacin & 23 & 0 & - & - & - & - & - & - \\
\hline & Ofloxacin & 351 & 0 & - & - & - & - & - & - \\
\hline \multicolumn{4}{|c|}{ Duration of FQs (per 1 day increment) } & 0.92 & $(0.77-1.1)$ & 0.3679 & 0.93 & $(0.78-1.1)$ & 0.3905 \\
\hline \multirow[t]{3}{*}{ Age } & $<8$ & 933 & 0 & - & - & - & - & - & - \\
\hline & $8-12$ & 501 & $3(0.6)$ & 3.2 & $(0.86-11.87)$ & 0.0816 & 2.98 & $(0.79-11.18)$ & 0.1054 \\
\hline & $\geq 12$ & 4,795 & $9(0.19)$ & \multicolumn{3}{|c|}{ Ref. } & & & \\
\hline
\end{tabular}

aORs, adjusted for age, severity, sex, cardiovascular diseases, congenital respiratory diseases, neuromuscular diseases, genetic metabolic diseases, neoplasms, autoimmune disease, and transplantation. AT, occurrence of Achilles tendinopathy.

line drugs in children under eight years of age. However, there are no evidence-based guidelines.

The FQs are generally well-tolerated, but they damaged the cartilage in the weight-bearing joints of juvenile animals (16). The pathophysiology of FQ-induced tendinopathy is still largely unknown. Various pathogenetic mechanisms have been postulated for arthropathies, such as the inhibition of collagen and glycosaminoglycans synthesis; the inhibition of mitochondrial function, resulting in the generation of free radicals; and oxidative stress, or the chelation of magnesium ions, all culminating in cartilage and tendon damage $(17,18)$. Animal models showed an increase in matrix-degrading activity and a decrease in matrix synthesis and fibroblast cell proliferation, thereby weakening the tendon and increasing the risk of rupture (19). FQ-induced tendinopathy has been reported in the literature and its incidence was estimated to be 0.14 $0.4 \%$ (20). FQ-induced tendinopathy preferentially occurs in adolescents during their pubertal growth spurt following long-term treatment. The time to the development of arthropathy ranged from $15 \mathrm{~min}$ to 30 days (median six days) after the administration of FQs (21). Achilles tendon rupture is also a well-known complication associated with FQs in elderly patients with preceding steroid use or underlying disease, but there have been no reports in healthy children to date (22).

On May 12, 2016, the U.S. Food and Drug Administration (FDA) advised restricting FQ antibiotic use for certain uncomplicated infections due to serious adverse reactions, such as tendinitis, tendon rupture, the worsening of myasthenia gravis, the risk of peripheral neuropathy and central nervous system effects, dermatologic, cardiac, and hypersensitivity reactions (23). The black box warning (FDA's strongest warning) for the drug was updated on July 26, 2016, for all oral and injectable FQ antibiotics due to side effects, such as disabling and potentially permanent effects on tendons, muscles, joints, nerves, and the central nervous system (24). Ciprofloxacin, gemifloxacin, levofloxacin, lomefloxacin, moxifloxacin, norfloxacin, ofloxacin, and tosufloxacin are currently available Korean FDA-approved FQs, but none of them have been approved by the Korean FDA for children under 18 years of age. However, FQ use has been expanded off-label to pediatric patients with conditions for which no alternative treatment 
was possible because of bacterial resistance or because other active antibiotics could not be given orally. Although FQs are not recommended as a first-line antibiotic because they have age-related contraindications, their use may be required in cases where macrolide resistance is suspected. Our study showed that $0.28 \%$ of childhood pneumonia was treated by the off-label use of FQs in real-world practice.

Increasing cases of FQ-associated tendinopathy have been reported since the first description of norfloxacin-associated Achilles tendinitis was published in 1983 (25). However, studies for children exposed to FQ have not clearly demonstrated the development of lesions consistent with those observed in animals $(26,27)$, and all musculoskeletal adverse effects reported in the literature were reversible following withdrawal of the treatment (28).

In a prospective study in France, among 276 children exposed to FQs, musculoskeletal adverse events occurred more frequently in FQs-treated children than in children not given FQ therapy (29). The Levaquin Pediatric Program phase three trials to assess the efficacy and safety of levofloxacin in children identified more musculoskeletal adverse events (arthralgia, arthritis, gait abnormality, or tendinopathy) in levofloxacin-treated children than in children not given FQ therapy (OR 2.4, 95\% CI: 1.1-5.2 at two months; OR 1.9; 95\% CI: $1.1-3.5$ at one year) (30). In contrast, FQ-treated children with cystic fibrosis consistently showed a low risk for arthropathy $(26,31)$. A healthcare claims study in the USA involving 7,897 children who had been prescribed any FQs reported no risk of tendon or joint disorders (TJD) with a $<1 \%$ incidence within 60 days after the first prescription of FQs (27).

Among the FQs, levofloxacin and ofloxacin seem to be associated with a higher risk of tendon damage relative to other FQs, and tendinitis-risk seems to be dependent upon the dose and duration of use (32). However, a systematic review of ciprofloxacin safety in those under 17 years of age did not find dose- and duration-dependent relationships in humans (28). Our study also showed no duration-dependent response.

The knowledge of this association is critical to ensuring timely diagnosis and management of these adverse events. FQ-induced tendinitis is distinguished from other forms of tendinitis by its abrupt-onset of sharp pain that occurs spontaneously upon walking or palpation, and substantial swelling (33). Early diagnosis and management are required and treatment consists of immediate discontinuation of the FQs with placement of the tendon at rest. Edema may persist from weeks to several months. The cardinal sign of tendinitis is tenderness and pain on movement or at rest, whereas the pain of tendon rupture is sudden. New or worsening pain in a patient on an FQ should lead the clinician to consider the possibility of tendinopathy. Therefore, clinicians should pay attention to joint pain, and switch to alternative treatments when joint pain occurs during FQ use. FQ can increase the susceptibility to further injury and should be ceased immediately when tendinopathy is suspected (23).

Compared to previous studies, the strengths of our study are as follows. The large sample size facilitated the analysis of the relationship between the use of FQs and the risk of AT and provided generalized information. Similar to our study, Yee et al. (27) demonstrated no relationship between the use of FQs and the risk of tendinopathy. However, they did not consider both the severity of the disease and baseline underlying diseases in the estimation models, whereas our study did. Furthermore, we considered longitudinally observed measurements of AT. For longitudinal data analysis, within-subject covariates are robust against between-subject confounders. We also considered sandwich estimators, which are robust for choosing variancecovariance structure among longitudinally observed measurements for each subject. Our study considered preexisting diseases and pneumonia severity as confounders by indication, and controlled their influence on the interpretation of our results.

Despite these contributions, some limitations should be considered in interpreting our findings. First, our study was based on the use of claims data. Because radiological and medical records are not available from the NHISS, the diagnostic reliability and validity of AT can be of concern. However, it is notable that AT caused by traffic accidents has not been covered by national health insurance. Therefore, no accidental AT cases could be included in these analyses. Second, mild symptoms of AT may not be reflected. Lastly, a certain number of potential confounders, such as a family history of musculoskeletal diseases, and personal status for nutrition, could not be used because they were not available in the NHISS.

Nonetheless, our results showed that the association between the use of FQs and AT was negligible in children under eight years of age. Rather, some underlying diseases may increase the risk of tendinopathy. Therefore, physicians could consider using FQs as a second-line option in the treatment of childhood pneumonia when there are no alternative therapeutic options. 


\section{Acknowledgments}

We would like to thank the officials and researchers in the National Health Insurance Sharing Service (NHISS) for their cooperation in constructing dataset. This manuscript was proofread and edited by the professional English editors at HARRISCO.

Funding: This research was supported by a grant from the Korea Health Technology R\&D Project through the Korea Health Industry Development Institute (KHIDI) funded by the Ministry of Health \& Welfare, Republic of Korea (grant number: HI16C2300). It was also supported by the Soonchunhyang University Research Fund. The funding source had no influence on the results reported here.

\section{Footnote}

Reporting Checklist: The authors have completed the STROBE reporting Checklist. Available at http://dx.doi. org/10.21037/jtd-20-2256

Data Sharing Statement: Available at http://dx.doi. org/10.21037/jtd-20-2256

Conflicts of Interest: All authors have completed the ICMJE uniform disclosure form (available at http://dx.doi. org/10.21037/jtd-20-2256). The authors have no conflicts of interest to declare.

Ethical Statement: The authors are accountable for all aspects of the work in ensuring that questions related to the accuracy or integrity of any part of the work are appropriately investigated and resolved. The study was conducted in accordance with the Declaration of Helsinki (as revised in 2013). The research protocol for this study was approved by the Ethical Review Board of Soonchunhyang University Seoul Hospital (approval number: SCHUH 2017-06-006). The requirement for informed consent was waived due to the retrospective nature of the study.

Open Access Statement: This is an Open Access article distributed in accordance with the Creative Commons Attribution-NonCommercial-NoDerivs 4.0 International License (CC BY-NC-ND 4.0), which permits the noncommercial replication and distribution of the article with the strict proviso that no changes or edits are made and the original work is properly cited (including links to both the formal publication through the relevant DOI and the license).
See: https://creativecommons.org/licenses/by-nc-nd/4.0/.

\section{References}

1. Ferwerda A, Moll HA, de Groot R. Respiratory tract infections by Mycoplasma pneumoniae in children: a review of diagnostic and therapeutic measures. Eur J Pediatr 2001;160:483-91.

2. Bébéar C, Pereyre S, Peuchant O. Mycoplasma pneumoniae: susceptibility and resistance to antibiotics. Future Microbiol 2011;6:423-31.

3. Saegeman V, Proesmans M, Dumke R. Management of macrolide-resistant Mycoplasma pneumoniae infection. Pediatr Infect Dis J 2012;31:1210-1.

4. Tanaka T, Oishi T, Miyata I, et al. Macrolide-Resistant Mycoplasma pneumoniae Infection, Japan, 2008-2015. Emerg Infect Dis 2017;23:1703-6.

5. Yamazaki T, Kenri T. Epidemiology of Mycoplasma pneumoniae Infections in Japan and Therapeutic Strategies for Macrolide-Resistant M. pneumoniae. Front Microbiol 2016;7:693.

6. Lung DC, Lam DSY, Chan E, et al. Practice recommendations for management of community acquired pneumonia in children. Hong Kong J Paediatr 2016;21:178-93.

7. Lee CH, Kim J, Jang EJ, et al. Healthcare utilisation by pregnant patients with asthma in South Korea: a cohort study using nationwide claims data. BMJ Open 2015;5:e008416.

8. Zhou Y, Zhang Y, Sheng Y, et al. More complications occur in macrolide-resistant than in macrolide-sensitive Mycoplasma pneumoniae pneumonia. Antimicrob Agents Chemother 2014;58:1034-8.

9. Izumikawa K. Clinical Features of Severe or Fatal Mycoplasma pneumoniae Pneumonia. Front Microbiol 2016;7:800.

10. Khoury T, Sviri S, Rmeileh AA, et al. Increased rates of intensive care unit admission in patients with Mycoplasma pneumoniae: a retrospective study. Clin Microbiol Infect 2016;22:711-4.

11. Mandell LA, Wunderink RG, Anzueto A, et al. Infectious Diseases Society of America/American Thoracic Society consensus guidelines on the management of communityacquired pneumonia in adults. Clin Infect Dis 2007;44 Suppl 2:S27-72.

12. Principi N, Esposito S. Macrolide-resistant Mycoplasma pneumoniae: its role in respiratory infection. J Antimicrob Chemother 2013;68:506-11. 
13. Miyashita N, Akaike H, Teranishi H, et al. Macrolideresistant Mycoplasma pneumoniae pneumonia in adolescents and adults: clinical findings, drug susceptibility, and therapeutic efficacy. Antimicrob Agents Chemother 2013;57:5181-5.

14. Kawai Y, Miyashita N, Kubo M, et al. Therapeutic efficacy of macrolides, minocycline, and tosufloxacin against macrolide-resistant Mycoplasma pneumoniae pneumonia in pediatric patients. Antimicrob Agents Chemother 2013;57:2252-8.

15. Bradley JS, Byington CL, Shah SS, et al. The management of community-acquired pneumonia in infants and children older than 3 months of age: clinical practice guidelines by the Pediatric Infectious Diseases Society and the Infectious Diseases Society of America. Clin Infect Dis 2011;53:e25-76.

16. Gough A, Barsoum NJ, Mitchell L, et al. Juvenile canine drug-induced arthropathy: clinicopathological studies on articular lesions caused by oxolinic and pipemidic acids. Toxicol Appl Pharmacol 1979;51:177-87.

17. Menschik M, Neumuller J, Steiner CW, et al. Effects of ciprofloxacin and ofloxacin on adult human cartilage in vitro. Antimicrob Agents Chemother 1997;41:2562-5.

18. Simonin MA, Gegout-Pottie P, Minn A, et al. Pefloxacininduced achilles tendon toxicity in rodents: biochemical changes in proteoglycan synthesis and oxidative damage to collagen. Antimicrob Agents Chemother 2000;44:867-72.

19. Williams RJ, 3rd, Attia E, Wickiewicz TL, et al. The effect of ciprofloxacin on tendon, paratenon, and capsular fibroblast metabolism. Am J Sports Med 2000;28:364-9.

20. Mehlhorn AJ, Brown DA. Safety concerns with fluoroquinolones. Ann Pharmacother 2007;41:1859-66.

21. Karande S, Kshirsagar NA. Ciprofloxacin use: acute arthropathy and long-term follow up. Indian Pediatr 1996;33:910-6.

22. Jackson MA, Schutze GE; COMMITTEE ON INFECTIOUS DISEASES. The Use of Systemic and Topical Fluoroquinolones. Pediatrics 2016;138:e20162706. 23. FDA. Maryland: FDA Drug Safety Communication:

Cite this article as: Kim Y, Park GW, Kim S, Moon HJ, Won S, Chung W, Yang HJ. Fluoroquinolone and no risk of Achilles-tendinopathy in childhood pneumonia under eight years of age - a nationwide retrospective cohort. J Thorac Dis 2021;13(6):3399-3408. doi: 10.21037/jtd-20-2256
FDA advises restricting fluoroquinolone antibiotic use for certain uncomplicated infections; warns about disabling side effects that can occur together. 2018 [cited 2018 Dec12]. Available online: https://www.fda.gov/ media/97602/download

24. FDA. Maryland: FDA drug safety communication: FDA updates warnings for oral and injectable fluoroquinolone antibiotics due to disabling side effects. C2018 [cited 2018 Dec12]. Available online: https://www.fda.gov/ media/119537/download

25. Bailey RR, Kirk JA, Peddie BA. Norfloxacin-induced rheumatic disease. N Z Med J 1983;96:590.

26. Burkhardt JE, Walterspiel JN, Schaad UB. Quinolone arthropathy in animals versus children. Clin Infect Dis 1997;25:1196-204.

27. Yee CL, Duffy C, Gerbino PG, et al. Tendon or joint disorders in children after treatment with fluoroquinolones or azithromycin. Pediatr Infect Dis J 2002;21:525-9.

28. Adefurin A, Sammons H, Jacqz-Aigrain E, et al. Ciprofloxacin safety in paediatrics: a systematic review. Arch Dis Child 2011;96:874-80.

29. Chalumeau M, Tonnelier S, D'Athis P, et al.

Fluoroquinolone safety in pediatric patients: a prospective, multicenter, comparative cohort study in France. Pediatrics 2003;111:e714-9.

30. Noel GJ, Bradley JS, Kauffman RE, et al. Comparative safety profile of levofloxacin in 2523 children with a focus on four specific musculoskeletal disorders. Pediatr Infect Dis J 2007;26:879-91.

31. Chyský V, Kapila K, Hullmann R, et al. Safety of ciprofloxacin in children: worldwide clinical experience based on compassionate use. Emphasis on joint evaluation. Infection 1991;19:289-96.

32. Bidell MR, Lodise TP. Fluoroquinolone-Associated Tendinopathy: Does Levofloxacin Pose the Greatest Risk? Pharmacotherapy 2016;36:679-93.

33. Zabraniecki L, Negrier I, Vergne P, et al. Fluoroquinolone induced tendinopathy: report of 6 cases. J Rheumatol 1996;23:516-20. 


\section{Supplementary}

Table S1 Types, categorization, classifications, and Anatomical Therapeutic Chemical (ATC) classification system codes, and Korea Food \& Drug Administration drug classification numbers of study medications

\begin{tabular}{|c|c|c|c|}
\hline Type of medications & Classification & ATC codes* & KFDA-DCN ${ }^{\dagger}$ \\
\hline \multirow{11}{*}{ Fluoroquinolones } & Ciprofloxacin & J01MA02 & $\begin{array}{l}\text { 134103ATB, 134105ATB, 134109ATB, 134103ACH, 134108ATR, 134101BIJ, } \\
\text { 134104BIJ, 134106BIJ, 134133BIJ, 134134BIJ, 134135BIJ }\end{array}$ \\
\hline & Pefloxacin & J01MA03 & 209501ATB \\
\hline & Lomefloxacin & J01MA07 & 184901ATB, 184903ATB, 184904 ATB, $184901 \mathrm{ACH}$ \\
\hline & Fleroxacin & J01MA08 & 159401ATB, 159402ATB \\
\hline & Sparfloxacin & J01MA09 & 230701ATB, 230702ATB \\
\hline & Moxifloxacin & J01MA14 & 380301ATB, 380302BIJ, 380335BIJ, 801601BIJ \\
\hline & Gemifloxacin & J01MA15 & 442901ATB, 442902BIJ \\
\hline & Gatifloxacin & J01MA16 & 434801ATB \\
\hline & Prulifloxacin & J01MA17 & 552901ATB \\
\hline & Pazufloxacin & J01MA18 & $566901 \mathrm{BIJ}$ \\
\hline & Garenoxacin & J01MA19 & 560301ATB \\
\hline
\end{tabular}

*, ATC codes: Anatomical Therapeutic Chemical (ATC) classifications system codes; ${ }^{\dagger}$, KFDA-DCN: Korea Food \& Drug Administration drug classification numbers.

Table S2 Definition of severe pneumonia based on the Solutions for Korean Medical Fee Contract System

\begin{tabular}{ll}
\hline & Codes in Korean Medical Fee Contract System \\
\hline Chest CT & HA424 or HA434 or HA464 or HA474 or HA444 \\
Oxygen supply & M0040 \\
ICU admission & AJ0, AJ1, AJ2, AJ3, AJ4, AJ5, 19001, 19002, 19003, 19004, 19005, 19400, 19402, 19412, \\
& $19422,19442,19410,19420,19430,19450,19200,19202,19212,19222,19242,19210$, \\
& $19220,19230,19240,19250,19260,19280,19290,19300,19302,19312,19322,19342$, \\
& $19310,19320,19330,19340,19350,19360,19380,19390,19500,19510,19520,19530$, \\
Tracheal intubation & $1954-, 19550,19560,19580,19590$ \\
Ventilator care or cardiopulmonary resuscitation & M08XX, M58XX \\
\hline
\end{tabular}


Table S3 Diagnoses and their matched coding criteria based on the Korean Classification of Disease 6 (KCD-6) and the International Classification of Disease 10 codes (ICD-10)

\begin{tabular}{ll}
\hline Categories & ICD-10 \\
\hline Achilles tendinopathy & S86.08 or M76.6, not S86.00 \\
Cardiovascular diseases & I0X.X or I1X.X or I2X.X or I3X.X or I40.9 or I42.X or I45.X or I47.X or I48.X or I49.X or I50.X or I51.X or P29. \\
& X or I7X.X or Q2X.X or S06.X \\
Congenital respiratory diseases & E84.X or P26.8 or P26.9 or P27.X or P28.5 or Q32.X or Q33.X or Q34.X \\
Neuromusculoskeletal diseases & G04.X or G05.X or G06.X or G11.X or G12.X or G31.X or G37.X or G7X.X or G80.X or G8X.X or G90.X or \\
& G91.X or G92.X or G93.X or G94.X or G95.X or I61.X or I62.X or I63.X or I64.X or I67.X or I69.X or I82.X or \\
I84.X or Q00.X or Q01.X or Q02.X or Q03.X or Q04.X or T90.X \\
Genetic metabolic diseases & E03.X or E2X.X or E7X.X or E80.X or E81.X or E82.X or E83.X or E84.X or E85.X \\
Immunodeficiency & B20.4 or B24.X or B25.X or B37.1 or B37.5 or B37.7 or B59.X or D8X.X \\
Neoplasms & C2X.X or C3X.X or C4X.X or C5X.X or C6X.X or C7X.X or C8X.X or C9X.X or D03.X or D05.X or D38.X or \\
Autoimmune diseases & D4X.X or D76.X or Z51.X or Z54.X \\
Transplant and complications & Z06.X or M08.X or M32.9 or M33.0 or M33.1 or M35.0 or M35.9 \\
\hline
\end{tabular}

